\title{
Molecular mechanism of vSGLT inhibition by gneyulin reveals antiseptic properties against multidrug-resistant gram-negative bacteria
}

\author{
Daniel Wiczew ${ }^{1} \cdot$ Anna Borowska $^{1} \cdot$ Kinga Szkaradek $^{1} \cdot$ Tomasz Biegus $^{1} \cdot$ Kamil Wozniak $^{1} \cdot$ Marcelina Pyclik $^{1}$. \\ Magdalena Sitarska ${ }^{1} \cdot$ Lukasz Jaszewski $^{1} \cdot$ Lukasz Radosinski $^{2} \cdot$ Beata Hanus-Lorenz $^{3} \cdot$ Sebastian Kraszewski $^{3}$ (I)
}

Received: 30 October 2018 / Accepted: 22 May 2019 / Published online: 11 June 2019

(C) The Author(s) 2019

\begin{abstract}
Faced with the worldwide spread of multidrug-resistant (MDR) bacterial strains, together with a lack of any appropriate treatment, urgent steps to combat infectious diseases should be taken. Usually, bacterial components are studied to understand, by analogy, the functioning of human proteins. However, molecular data from bacteria gathered over the past decades provide a sound basis for the search for novel approaches in medical care. With this current work, we want to direct attention to inhibition of the vSGLT glucose transporter from Vibrio parahaemolyticus belonging to the sodium solute symporter (SSS) family, to block sugar transport into the bacterial cell and, as a consequence, to limit its growth. Potential bacteriostatic properties can be drawn from commercially available drugs developed for human diseases. This goal can also be reached with natural components from traditional herbal medicine. The presented data from the numerical analysis of 44 known inhibitors of sodium glucose symporters shed light on potential novel approaches in fighting Gram-negative multidrug-resistant microorganisms.
\end{abstract}

Keywords Multidrug-resistant gram-negative microorganisms $\cdot$ Bacterial resistance $\cdot$ Aseptic agents $\cdot$ vSGLT glucose transporter inhibition

\section{Introduction}

Microbial resistance to currently used antibiotics is reaching a critical level. Infections caused by multidrug-resistant microorganisms present daily challenges to physicians, clinicians,

This paper belongs to Topical Collection 8th conference on Modeling \& Design of Molecular Materials (MDMM 2018)

Electronic supplementary material The online version of this article (https://doi.org/10.1007/s00894-019-4073-9) contains supplementary material, which is available to authorized users.

Sebastian Kraszewski

sebastian.kraszewski@pwr.edu.pl

1 Faculty of Chemistry, Wroclaw University of Science and Technology, Norwida 4/6, 50-370 Wroclaw, Poland

2 Faculty of Chemistry, Division of Bioprocess and Biomedical Engineering, Wroclaw University of Science and Technology, Norwida 4/6, 50-370 Wroclaw, Poland

3 Faculty of Fundamental Problems of Technology, Department of Biomedical Engineering, Wroclaw University of Science and Technology, Wybrzeze Wyspianskiego 27, 50-370 Wroclaw, Poland and patients throughout the world. There have been attempts to tackle bacterial resistance to antimicrobial drugs by discovering new antibiotics and by chemical modification of existing drugs. Research into new antibiotics and novel mechanisms of action involves high development costs and provides a poor return on investments. According to a WHO analysis of new antibacterial products, (as of May 2017) 66 new substances are on the clinical development pathway [1], and these show hopeful signs. Among these substances are antibiotics (including multidrug combinations) and biological agents active against only specific pathogens or a limited number of resistant strains. Almost all these agents are modifications of already existing antibiotic classes. For the most part, they already address specific and well-known resistance mechanisms. Highly disquieting is the information about a lack of potential treatment options for multidrug-resistant Gram-negative (MDRGN) bacteria (e.g., Pseudomonas aeruginosa, Acinetobacter baumannii, Enterobacteriaceae). To combat MDRGN bacteria, we need to find molecules with certain properties to shatter resistance mechanisms, to disturb proliferation processes leading to metabolic imbalance, to enact physical destruction of cellular structures or to limit cell 
growth. Some strategies are focused on screening drugs currently accessible on the market to identify side effects that may be desirable in terms of antimicrobial development, thus not incurring the costs of introducing a new drug. Another strategy is to screen a large number of natural molecules present in plants on the basis of evidence from alternative medicine treatment of infections. Ultimately, the solution may be the starving of single cells, as they are complete bacterial organisms, by preventing them from taking nutrients from the environment. For this purpose, already known molecular mechanisms can be exploited.

The active transport of many substrates is driven by the electrochemical gradient across the plasma membrane through transmembrane proteins from the sodium solute symporter (SSS) family [2]. Solute sodium symporters (SSS) that transport sugars, vitamins, amino acids or inorganic ions according to the $\mathrm{Na}^{+}$gradient across cell membranes are a large family of proteins sharing a similar core architecture across different genes [3]. One currently studied $\mathrm{Na}^{+}$-driven symport is a sodium/galactose transporter from Vibrio parahaemolyticus (vSGLT) used as a structural and functional homolog for human SGLT1 and SGLT2 transporters involved in diabetes treatment $[4,5]$. The recently resolved crystal structure of the vSGLT protein opened new possibilities for drug discoveries (medicines for humans) based on molecular mechanisms. Despite these applications, we see another and far more prominent way of studying native bacterial transporter functioning for the development of medicines for antibacterial treatment. Here, we propose to reveal a molecular mechanism useful for inhibition of bacterial nourishment uptake, based on plant-derived phlorizin molecules and their synthetic derivatives, as well as on a set of natural SGLT inhibitors. Phlorizin is known to be at least a 1000 -fold weaker inhibitor for bacterial vSGLT than for human intestinal brush border SGLT1 [6], probably due to the absence in vSGLT of the supposed phlorizin binding domain [7]. Hence, there is a need to understand the molecular mechanism of $\mathrm{Na}^{+}$dependent galactose transport inhibition.

\section{Methods}

As a representative of SSS we chose the galactose symporter vSGLT from Vibrio parahaemolyticus originally crystalized by Faham et al. with a resolution of $2.7 \AA$ (PDB code: 3DH4) [8]. As shown in Fig. 1, both vSGLT structures (generally open and closed) were modeled from M1 to W543 as a K294A, C411A, A423C inactive mutant, using the program MODELLER 9.20 [9] following the protocol described in [8], to exclude the basic hydrogen interaction from K294 with the sugar molecule (necessary for effective transport) and in this way we found a strong inhibitory bonding in other, sodium-independent, locations. The inward open structure (vSio) was homology modeled
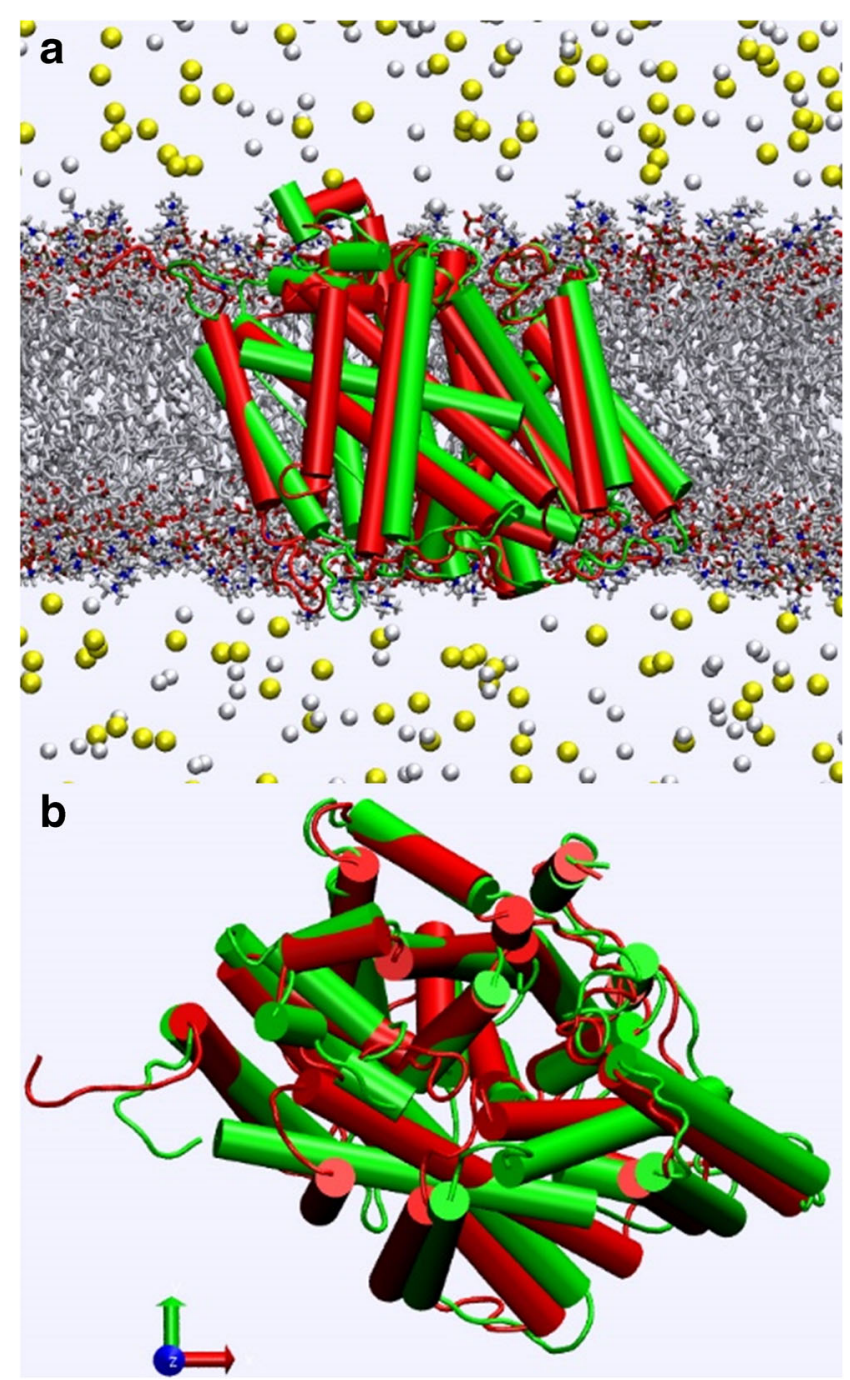

Fig. 1 Closed channel (vSio - red), and open channel (vSoo - green) superimposed structures, embedded in lipidic membrane, a side view (a) and top view (b). vSGLT symporter structures were modeled from M1 to W543 amino acids on 3DH4.pdb (close conformation) and on 2XQ2.pdb (open conformation) templates, respectively

directly on a template structure provided by Faham et al. [8], while the open outward structure (vSoo) was modeled directly on an open-outward K294A mutant structure provided by Watanabe et al. (PDB code: 2XQ2) [5]. Both prepared models, $\mathrm{vSio}$ and vSoo, respectively, were next used to perform molecular dynamics simulations in order to test structural stability and to get protein conformations for further docking analyses. First, both molecular models were reconstructed separately within 604 zwitterionic palmitoyloleoylphosphatidylcholine (POPC) pre-equilibrated lipids (using Charmm-GUI web tool [10]) forming a membrane patch, 56,415 TIP3 water molecules, $162 \mathrm{Na}^{+}$and $159 \mathrm{Cl}^{-}$counterions ensuring $0.15 \mathrm{moll}$ concentration, giving a final volume of $2500 \mathrm{~nm}^{3}$. We decided to use a POPC membrane since this approach is very often used in MD simulations, does not introduce an additional surface charge, and has no important impact on the studied active 
site of vSGLT buried deep into the protein structure. Both systems (vSio and vSoo) were run for 20 ns of equilibration followed by a $100 \mathrm{~ns}$ production run using NAMD 2.9 software [11] with a CHARMM27 force field [12], with electrostatics treated by PME, barostat set to $1 \mathrm{~atm}$, thermostat set to $300 \mathrm{~K}$, and hydrogen bonds treated with SHAKE algorithm. The RMSD of both trajectories are shown in Fig. S1 in Supplementary Materials. Secondly, from each of two $100 \mathrm{~ns}$ production runs we extracted 2000 configurations (snapshots) of the protein itself (without lipids, water, and ions) in vSio and 2000 configurations in vSoo states, respectively.

Potential inhibitors of SSS transporters were chosen across several synthetic glucoside analogs, based on the first plantderived SGLT inhibitor, phlorizin [13], which are now commercially available as drugs in diabetes treatment. Others were synthetic molecules with some SGLT inhibitory activity, which were not accepted in clinical trials. Following the Chang-Ik Choi review, we decided to also include a set of natural active compounds (named further $\mathbf{N x x}$ after ChangIk Choi) that are present in products of traditional herbal medicine [14]. For structural details on the 44 chosen inhibitors, please see Supplementary Materials, Table S1. Numerical models of inhibitors were drawn and optimized at a quantum level (consisting on MM-MM3_Geo followed by MO-GPM6_H2O_Geo procedures) using SCIGRESS 3.3.3 software [15]. Next, prepared inhibitor models were flexibly docked on two sets (vSoo and vSio) of 2000 configurations (snapshots) of the vSGLT protein. The ligands were consecutively docked on each set of snapshots using Autodock VINA software [16], with an embedded energy estimating algorithm (no further improvements were done). This resulted in 40,000 docking poses for each inhibitor, which were further statistically analyzed. The search area for docking was restricted to only the extracellular side of the pore channel. This approach, recently described by Gioia et al. [17], permits the consideration of protein dynamics, importantly saving computational resources.

\section{Results and discussion}

Since vSGLT dynamics alternates between the outward open (vSoo) and the inward open (vSio) conformations across six structural states, we decided to test interactions between vSGLT and its potential inhibitors at the extracellular side of the protein only, but on both vSoo and vSio states, respectively. This approach permitted us to mimic the ligand incursion from the external environment, since the chosen molecules cannot freely pass through the membrane. Thus, testing intracellular interactions seems pointless. Performing molecular dynamics for 44 ligands with two different states of receptor is a very challenging task. Hence, we chose a theoretical approach combining molecular dynamics and dynamic docking.
The results from massive docking of ligands on vSio and vSoo structures of vSGLT are presented in Fig. 2 as normalized histograms of binding energies. Each curve represents a binding energy distribution over 40,000 collected docking poses for each inhibitor. Galactose, a native vSGLT substrate, as well as glucose, represents interaction energies of $-4.9 \mathrm{kcal} \mathrm{mol}^{-1}$ and $-5.1 \mathrm{kcal} \mathrm{mol}^{-1}$ with closed (inward-open) and open (outward-open) channels, respectively. Their interactions, in the context of protein dynamics and taken indirectly into account in 2000 protein configurations from molecular dynamics trajectories, represent a relatively narrow distribution, which can be interpreted as a highly selective interaction.

Analyzing the peaks of binding energy distributions, one can notice that all distributions are close to Gaussian shaped, which reflects the normal distribution behavior of the genetic algorithm for search poses implemented in VINA software. Thus, it is reasonable to define a full width at half maximum (FWHM), which we collected together with the peak positions in Table 1. Compared to inhibitors showing a broader binding energy distribution with an FWHM parameter close to $1.0 \mathrm{kcal}$ $\mathrm{mol}^{-1}$, all galactose derivatives (i.e., glucose and two forms of ascorbic acid — vitamin C) present FWHM at $0.5 \mathrm{kcal} \mathrm{mol}^{-1}$ level, confirming the existence of a selective binding site particularly designed for the galactose molecule. Even the phlorizin molecule, the first discovered natural inhibitor of SGLT channels, has an energy distribution twice as broad as that of galactose. Its potency for SGLT inhibition is, however, visible in much stronger mean energy interactions, reaching $-7.8 \mathrm{kcal} \mathrm{mol}^{-1}$ for closed and $-7.4 \mathrm{kcal} \mathrm{mol}^{-1}$ for open channels, respectively. These data suggest that phlorizin can easily interact with the SGLT channel regardless of the channel state. None of the synthetic O- and C-glucoside derivatives show stronger (more negative) mean binding energies than phlorizin for closed channels (vSio), ranging from $-6.4 \mathrm{kcal} \mathrm{mol}^{-1}$ for sergliflozin (O-glucoside) up to $-7.1 \mathrm{kcal} \mathrm{mol}^{-1}$ for YM-543 (C-glucoside). For open state channels (vSoo), there are already eight synthetic derivatives with more negative binding energy than is represented by phlorizin. Two of these, canagliflozin and YM-543 (both C-glucosides), even exceed the value of $-8.0 \mathrm{kcal} \mathrm{mol}^{-1}$. This is an energy-based reason for the better inhibition potency of several phlorizin derivatives available on the market.

Regarding the analyzed set of natural compounds, the situation looks similar at first glance. In the case of closed channel structures (vSio), only two products interact more strongly than phlorizin (i.e., N18 and N19). For open channel structures (vSoo), there are already nine molecules more potent than phlorizin: N02, N06, N07, N08, N11, N14, N17, N18, and N19. Two of these have even stronger interactions that are one kcal mol ${ }^{-1}$ more than phlorizin, N18 and N19, as in the case of closed channel vSio state, with better prediction for N19. Both molecules, known under the names gneyulin A and gneyulin B, can be extracted from Gnetum gnemonoides, a 
Fig. 2 Distributions of binding energies of inhibitors interacting with vSGLT closed (a) and open (b) channel structures


kind of tropical lianas. Substances isolated from Gnetum species, belonging to the group of stilbenes, are already shown to reveal antioxidant, antimicrobial, and some inhibitory activities [18].

Taking a closer look at the molecular complexes of gneyulin B (N19), phlorizin and galactose with vSGLT channels, we verified their docking poses, but only those having energy interactions around the peak position from its energy interaction distribution (see Fig. 2), as the most probable configurations. The specific interactions observed in the six ligand-protein complexes (two states of vSGLT and three ligands) involving contacts with specific amino acids are summarized in Table 2. Inward open vSGLT configuration presents three distinct active sites for the studied galactose, N19 and phlorizin molecules. In Table 2, we distinguish these by colors corresponding to those of the amino acids from Fig. S2 in Supplementary Materials. At the first interaction site (colored green) involved amino acids (S9, D12, T156, I157, L158, D323, I324, Q344, V348, and K351) are located in the external mouth of the glucose pore. At this location, N19 presents very similar binding distances to phlorizin, but their interaction profiles show more distant interactions than those for galactose, suggesting that their functioning as a chaperone molecule may not be the key mechanism of vSGLT inhibition. The second N19 and phlorizin interaction site (colored blue, see also Fig. 3a) involves amino acids from different loops between TM2e and TM3, between EL6 and TM7e, between TM10 and TM11, and from helix TM11. This location also shows a moderate specificity for galactose binding, with shorter minimal distances, which is to be expected because of the smaller size of galactose. S79, I80, N245, Q422, and Q425 linked together through binding with N19 or phlorizin may freeze the channel in closed conformation. Xie et al. point out that Q425C mutation of vSGLT is sensitive to MTSEA (2aminoethyl methanethiosulfonate) binding, which results in modified transport of $\mathrm{Na}^{+} /$glucose transport activity. As suggested, Q425 thus plays a critical role in glucose binding/ translocation [6]. The third interaction site (colored red) is 
Table 1 Results of Gaussian fits on binding energy distributions of over 40,000 collected docking poses for each inhibitor

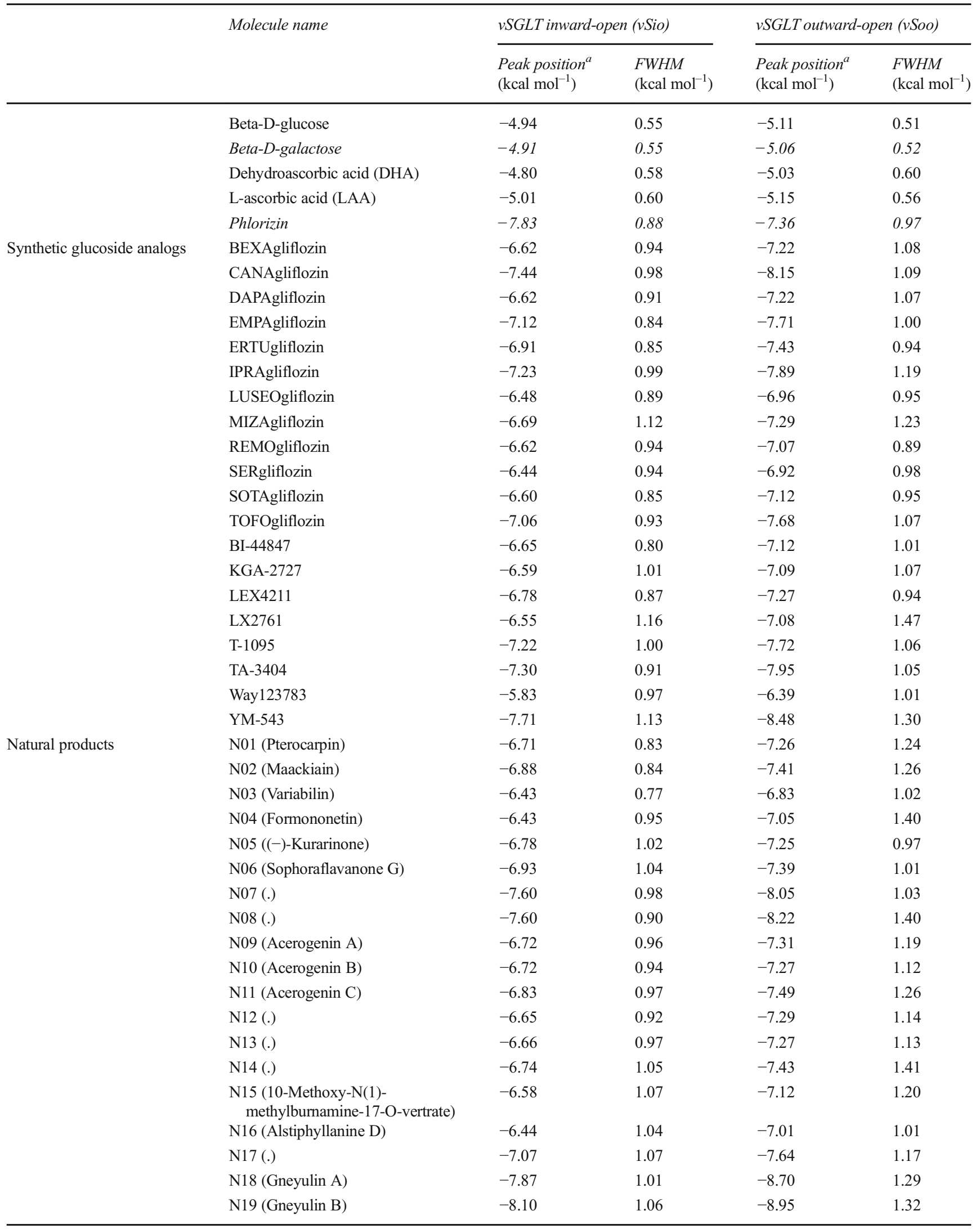

${ }^{\text {a }}$ Standard error not exceeding $0.009 \mathrm{kcal} \mathrm{mol}^{-1}$ 
more important for N19, with four amino acids involved (N328, N334, A335, K337), than for phlorizin, which, if it interacts there, optimizes mostly with N328 and A335 within the same loop between EL8a and EL8b helices. This location is almost absent for galactose and should not be considered as possible for it. Sites colored green and blue are indirectly involved with gating helices TM5 and TM10, providing reasons to accept them as possible inhibition sites, in contrast to the red site. The green site is related to TM5 through I157 and L158 located at the loop before TM5, while the blue site is related with TM10 through Q422 located after TM10. We are convinced that these complicated interactions of N19, with mean binding energy at $-8.1 \mathrm{kcal} \mathrm{mol}^{-1}$, strongly perturb channel dynamics and sterically prevent the channel from changing to its outward-open state.

In contrast to vSio conformation, in open outward configuration of vSGLT, N19 and phlorizin show no similarities in interaction profiles compared to galactose (see Table 2). Phlorizin and N19 interact inside opened vSGLT pores with a large number of amino acids, with an advantage for N19. This could explain the stronger (more negative) interaction energy for N19 than for phlorizin. However, N19 is a larger molecule than phlorizin and one could expect its worse positioning to result in greater distances between interactions, which is not obviously the case (see Fig. 3b). Both molecules interact with Q69 and Q428, which were selected by point mutations as being crucial for $\mathrm{Na}^{+}$-dependent glucose transport [8]. Faham et al. also show their importance for galactose binding on the extracellular gate at M73, Y87, and F424 and in the binding site at Y263 and W264. In the case of vSoo conformation, the channel is still in a pre-openedwith-galactose-gate-closed state; thus, results for phlorizin and galactose shown in Table 2 are not well optimized with experimentally proved amino acids. However, in this

Table 2 Protein-ligand aliphatic sidechain minimal distances observed for conformation complexes of N19 phlorizin and galactose, for open (vSoo), and closed (vSio) channels

\begin{tabular}{|c|c|c|c|c|}
\hline & \multirow{2}{*}{$\begin{array}{l}\text { vSGLT amino acid residue } \\
\text { (amino acid location) }\end{array}$} & \multicolumn{3}{|c|}{ Minimal distance $[\AA ̊]$} \\
\hline & & N19 & Phlorizin & Galactose \\
\hline \multirow{19}{*}{ 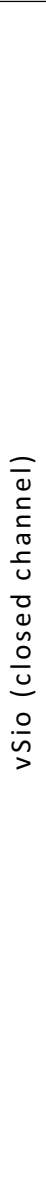 } & SER9 (N-terminal linear chain) & 1.2 & 1.1 & 0.3 \\
\hline & ASP12 (N-terminal linear chain) & 1.8 & 1.8 & 0.7 \\
\hline & THR156 (TM4 helix) & 1.8 & 1.4 & 1.5 \\
\hline & ILE157 (loop between TM4 and TM5) & 1.8 & 1.8 & 1.4 \\
\hline & LEU158 (loop between TM4 and TM5) & 1.8 & 1.8 & 1.3 \\
\hline & ASP323 (EL8a helix) & 1.3 & 1.1 & 1.7 \\
\hline & ILE324 (loop between EL8a and EL8b helices) & 1.1 & 1.0 & 1.6 \\
\hline & GLN344 (EL8b helix) & 1.3 & 1.2 & 0.2 \\
\hline & VAL348 (loop between EL8b and TM9 helices) & 1.4 & 1.0 & 0.5 \\
\hline & LYS351 (TM9 helix) & 1.2 & 1.0 & 0.3 \\
\hline & SER79 (loop between TM2e and TM3 helices) & 0.9 & 1.2 & 0.8 \\
\hline & ILE80 (loop between TM2e and TM3 helices) & 2.0 & 2.5 & 0.2 \\
\hline & ASN245 (loop between EL6 and TM7e helices) & 1.1 & 1.1 & 0.3 \\
\hline & GLN422 (loop between TM10 and TM11 helices) & 1.0 & 1.1 & 0.5 \\
\hline & GLN425 (TM11 helix) & 1.4 & 1.1 & 0.6 \\
\hline & ASN328 (loop between EL8a nad EL8b helices) & 1.1 & 0.9 & 0.5 \\
\hline & ASN334 (loop between EL8a nad EL8b helices) & 1.2 & 1.2 & 1.6 \\
\hline & ALA335 (loop between EL8a nad EL8b helices) & 1.0 & 1.3 & 0.8 \\
\hline & LYS337 (EL8b helix) & 0.8 & 1.0 & 0.4 \\
\hline
\end{tabular}


Table 2 (continued)

\begin{tabular}{|c|c|c|c|c|}
\hline \multirow{21}{*}{ 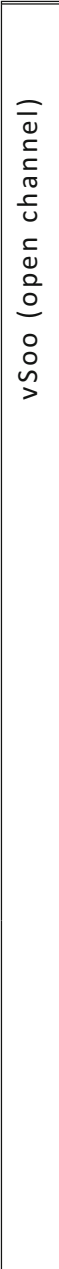 } & ASN64 (loop between TM2i and TM2e helices) & 1.0 & 1.2 & 1.1 \\
\hline & SER66 (loop between TM2i and TM2e helices) & 0.9 & 1.0 & 1.2 \\
\hline & GLU68 (TM2e helix) & 1.2 & 1.1 & 1.1 \\
\hline & GLN69 (TM2e helix) & 1.0 & 1.0 & 1.0 \\
\hline & MET73 (TM2e helix) & 1.8 & 1.7 & 1.1 \\
\hline & SER74 (TM2e helix) & 1.0 & 1.2 & 1.3 \\
\hline & GLY75 (TM2e helix) & 1.0 & 1.2 & 1.5 \\
\hline & TYR87 (TM3 helix) & 1.1 & 1.0 & 1.9 \\
\hline & GLU88 (TM3 helix) & 1.5 & 1.8 & 1.8 \\
\hline & ASN142 (TM4 helix) & 0.8 & 1.0 & 1.4 \\
\hline & SER145 (TM4 helix) & 0.9 & 1.0 & 1.2 \\
\hline & GLN242 (loop between EL6 and TM7e helices) & 1.0 & 1.5 & 1.7 \\
\hline & ASN245 (loop between EL6 and TM7e helices) & 1.1 & 1.1 & 1.3 \\
\hline & ASN260 (TM7e helix) & 1.0 & 0.9 & 1.0 \\
\hline & TYR263 (TM7e helix) & 1.0 & 1.0 & 1.3 \\
\hline & TRP264 (TM7e helix) & 0.9 & 0.9 & 0.9 \\
\hline & PHE424 (TM11 helix) & 1.0 & 1.3 & 1.5 \\
\hline & GLN425 (TM11 helix) & 0.7 & 1.0 & 1.3 \\
\hline & GLN428 (TM11 helix) & 0.9 & 1.0 & 1.3 \\
\hline & GLU429 (TM11 helix) & 1.1 & 1.6 & 1.8 \\
\hline & PHE479 (C-terminal linear chain) & 1.0 & 1.3 & 1.1 \\
\hline
\end{tabular}

Complexes taken for analysis have only the most probable interaction energies taken as a peaks from Fig. 1. The presented amino acids occurred in at least $50 \%$ of analyzed complexes. Strikethrough of the minimal values indicates that the amino acid enters into interactions in less than $20 \%$ of studied complexes for a given ligand. A cutoff of $1.5 \AA$ has been used. Minimal distance is defined as the distance between the closest atoms of a given amino acid and ligand, respectively. Colors represent different active sites, as shown in Supplementary Information Fig. S2

conformation N19 is already able to interact with all three, M73 (though quite poorly), Y87, and F424; yet, phlorizin optimizes interactions only with Y87, which may make this a weak inhibitor for vSGLT. Interactions with Y263 are identical for N19 and phlorizin, while with W264 all three compounds behave in the same manner. Our results also show shorter distances between interactions with E88 and Q428 residues in the case of compound N19 than for phlorizin, and following Raja and Kinne those two amino acids in the sugar binding pocket form a proper gate for sugar translocation [19]. We also found that none of the binding sites appear to interfere with the sodium binding site at the level of A62, I65, A361, S365, S364 [8]; although, N19 presents interaction lower than $1 \AA$ for S66, as it is in the close vicinity of sodium binding site. The multiple specific interactions with N19 described here may be the origins of vSGLT transporter inhibition.

\section{Conclusions}

In this study, various commercially available, synthetic, and natural active compounds were analyzed in the context of vSGLT Gram-negative bacteria galactose symporter inhibition. Although many molecular data concerning bacteria cells are available, recent studies have mainly been focused on understanding molecular mechanisms transferrable to human body aspects. There is still a lack of a wider view on possible applications of such data, especially toward current global problems, such as the spread of multidrug-resistant Gram-negative bacteria strains. We postulate that the interaction scheme described here for vSGLT inhibition can be successfully used for at least antiseptic strategies based on preventing microorganisms from uptake of nutrients.

We found that phlorizin interacts with inward open (vSio) and outward open (vSoo) channel structures with a slightly 


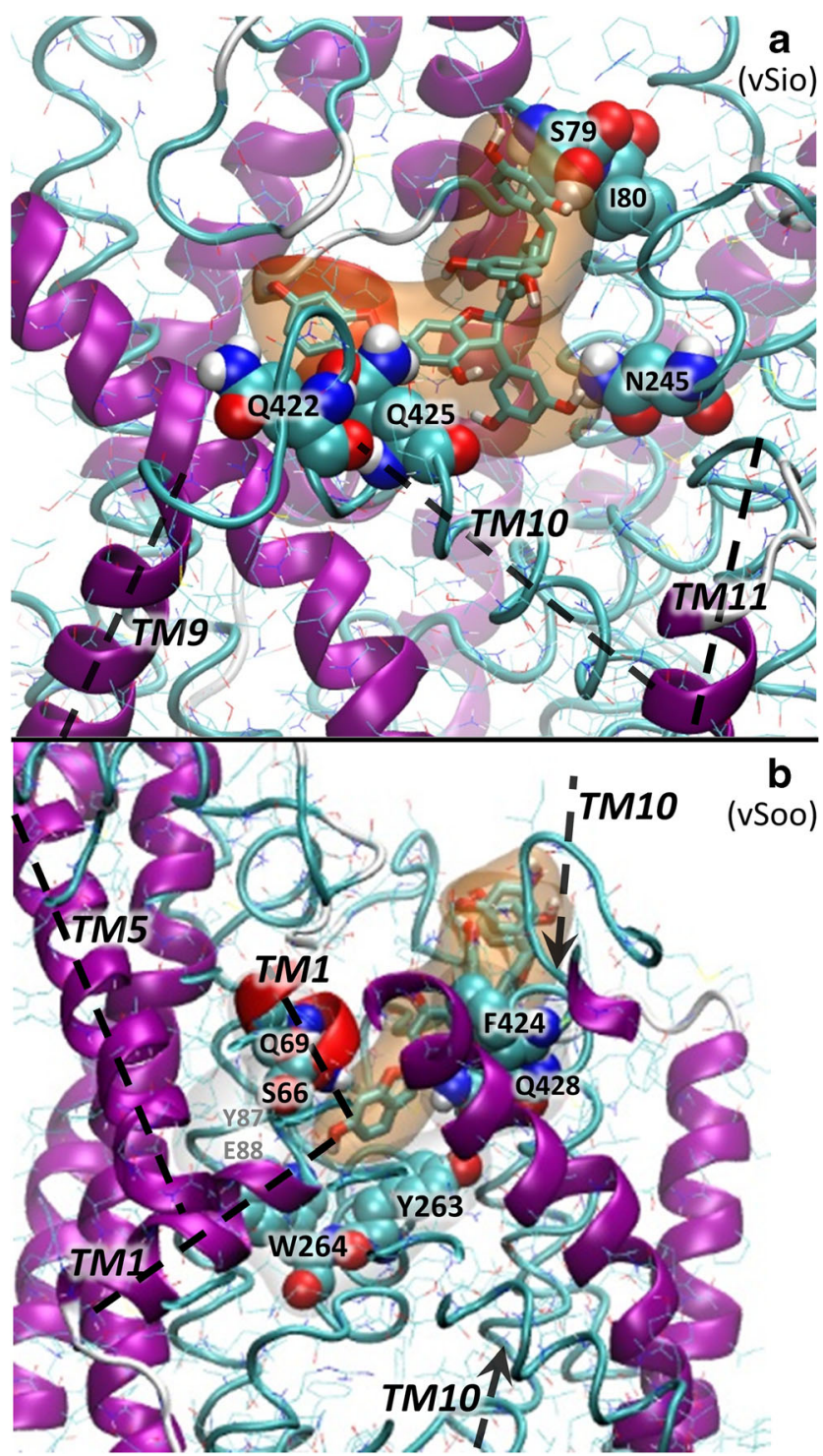

Fig. 3 The most representative interactions of natural compound N19 with closed (a) and open (b) vSGLT channel structures. Inset a shows N19 interactions in blue site only

stronger interaction to closed channels, thus suggesting that the observed experimentally weak inhibition potency may come from the impossibility of opening the channel to the conducting state, rather than occluding the opened pore, especially in the absence of a phlorizin binding domain in bacterial vSGLT. None of the synthetic derivatives of phlorizin show this ability, but they interact only with vSGLT channels in outward open conformation. In contrast to synthetic O- and C-glucosides, natural compounds studied here (N01-N19), besides in some cases presenting stronger interactions with vSGLT than phlorizin, the most potent inhibitor, show three specific interactions with closed galactose transporters (at pore entry, near the TM2E helix, and near the EL8b helix), and these possibly block the opening of the channel by hindering movements of gating helices TM5 and TM10. In open outward conformation of the transporter, N19 penetrates deeply into the galactose pathway interacting with Y263 and W264 amino acids involved in galactose stacking, with E88 and Q428, the gate for sugar translocation, and with the extracellular gate at M73, Y87, and F424 residues, thereby explaining possible molecular mechanisms constituting the origins of vSGLT inhibition. Taken together, our results highlight the importance of use of dedicated inhibitors to starving bacterial cells, and hence to combat resistant strains.

Acknowledgments Calculations have been carried out using resources provided by Wroclaw Centre for Networking and Supercomputing (http://wcss.pl), grant No. 274.

This study was partially financed by the Foundation for Polish Science; project HOMING PLUS (No. HOMING PLUS/2013-8/6).

This study was partially supported by a grant from the Polish National Science Center (NCN); project OPUS 10 (No. 2015/19/B/NZ7/02380).

Open Access This article is distributed under the terms of the Creative Commons Attribution 4.0 International License (http:// creativecommons.org/licenses/by/4.0/), which permits unrestricted use, distribution, and reproduction in any medium, provided you give appropriate credit to the original author(s) and the source, provide a link to the Creative Commons license, and indicate if changes were made.

\section{References}

1. World Health Organization (2017) Antibacterial agents in clinical development: an analysis of the antibacterial clinical development pipeline, including tuberculosis

2. Reizer J, Reizer A, Saier MH (1994) A functional superfamily of sodium/solute symporters. Biochim Biophys Acta Rev Biomembr 1197(2):133-166

3. Wright EM et al (2004) Surprising versatility of $\mathrm{Na}^{+}$-glucose cotransporters: SLC5. Physiology 19(6):370-376

4. Turk E et al (2000) Molecular characterization of vibrio parahaemolyticus vSGLT a model for sodium-coupled sugar cotransporters. J Biol Chem 275(33):25711-25716

5. Watanabe A et al (2010) The mechanism of sodium and substrate release from the binding pocket of vSGLT. Nature 468:988

6. Xie Z, Turk E, Wright EM (2000) Characterization of the vibrio parahaemolyticus $\mathrm{Na}^{+} /$glucose cotransporter: a bacterial member of the sodium/glucose transporter (SGLT) family. J Biol Chem 275(34):25959-25964

7. Raja M, Kinne RKH (2015) Identification of phlorizin binding domains in sodium-glucose cotransporter family: SGLT1 as a unique model system. Biochimie 115:187-193

8. Faham $\mathrm{S}$ et al (2008) The crystal structure of a sodium galactose transporter reveals mechanistic insights into $\mathrm{Na}^{+}$/sugar symport. Science (New York, NY) 321(5890):810-814

9. Webb B, Sali A (2014) Comparative protein structure modeling using MODELLER. Curr Protoc Bioinformatics 47(1):5.6. 1-5.6. 32

10. Jo $\mathrm{S}$ et al (2008) CHARMM-GUI: A web-based graphical user interface for CHARMM. J Comput Chem 29(11):1859-1865

11. Phillips JC et al (2005) Scalable molecular dynamics with NAMD. J Comput Chem 26(16):1781-1802

12. Vanommeslaeghe $\mathrm{K}$ et al (2010) CHARMM general force field: a force field for drug-like molecules compatible with the CHARMM 
all-atom additive biological force fields. J Comput Chem 31(4): 671-690

13. Ehrenkranz JRL et al (2005) Phlorizin: a review. Diabetes Metab Res Rev 21(1):31-38

14. Choi C-I (2016) Sodium-glucose cotransporter 2 (SGLT2) inhibitors from natural products: discovery of next-generation antihyperglycemic agents. Molecules 21(9):1136

15. SCIGRESS (2013) Fujitsu Limited, Tokyo

16. Trott O, Olson AJ (2010) AutoDock Vina: improving the speed and accuracy of docking with a new scoring function, efficient optimization, and multithreading. J Comput Chem 31(2):455-461

17. Gioia D et al (2017) Dynamic docking: a paradigm shift in computational drug discovery. Molecules 22(11):2029
18. Shimokawa Y et al (2010) Gneyulins A and B, trimers, and noidesols $\mathrm{A}$ and $\mathrm{B}$, dihydroflavonol-C-glucosides, from the bark of Gnetum gnemonoides. J Nat Prod 73(4):763-767

19. Raja M, Kinne RKH (2012) Structural insights into genetic variants of $\mathrm{Na}^{+}$/glucose cotransporter SGLT1 causing glucose-galactose malabsorption: vSGLT as a model structure. Cell Biochem Biophys 63(2):151-158

Publisher's note Springer Nature remains neutral with regard to jurisdictional claims in published maps and institutional affiliations. 